\title{
Black hole singularity resolution via the modified Raychaudhuri equation in loop quantum gravity
}

\author{
Keagan Blanchette, ${ }^{a}$ Saurya Das, ${ }^{b}$ Samantha Hergott, ${ }^{a}$ Saeed Rastgoo, ${ }^{a}$ \\ ${ }^{a}$ Department of Physics and Astronomy, York University \\ 4700 Keele Street, Toronto, Ontario M3J 1 P3 Canada \\ ${ }^{b}$ Theoretical Physics Group and Quantum Alberta, Department of Physics and Astronomy, \\ University of Lethbridge, 4401 University Drive, Lethhbridge, Alberta T1K 3M4, Canada \\ E-mail: kblanch@yorku.ca, saurya.das@uleth.ca, sherrgs@yorku.ca, \\ srastgoo@yorku.ca
}

ABSTRACT: We derive loop quantum gravity corrections to the Raychaudhuri equation in the interior of a Schwarzschild black hole and near the classical singularity. We show that the resulting effective equation implies defocusing of geodesics due to the appearance of repulsive terms. This prevents the formation of conjugate points, renders the singularity theorems inapplicable, and leads to the resolution of the singularity for this spacetime. 


\section{Contents}

1 Introduction $\quad 1$

2 Interior of the Schwarzschild black hole $\quad 3$

3 Classical dynamics $\quad 6$

3.1 Classical Hamiltonian and equations of motion 6

3.2 Classical Raychaudhuri equation $\quad 10$

4 Effective dynamics and Raychaudhuri equation $\quad 11$

$4.1 \stackrel{\mu}{\mu}$ scheme 14

$\begin{array}{lll}4.2 & \bar{\mu} \text { scheme } & 17\end{array}$

$\begin{array}{lll}4.3 & \bar{\mu}^{\prime} \text { scheme } & 19\end{array}$

5 Discussion and outlook $\quad 22$

\section{Introduction}

It is well known that General Relativity (GR) predicts that all reasonable spacetimes are singular, and therefore its own demise. While a similar situation in electrodynamics was resolved in quantum electrodynamics, quantum gravity has not been completely formulated yet. One of the primary challenges of candidate theories such as string theory and loop quantum gravity (LQG) is to find a way of resolving the singularities.

Singularities in GR are defined differently compared to other field theories. While curvature scalars (such as the Kretschmann scalar) approaching infinity (similar to the electric field diverging at the seat of a charge) is a strong indication of singularities, it is neither a necessary nor a sufficient condition for singularities in GR. The necessary and sufficient condition for a singular spacetime is the existence of a set of geodesics which begin and/or end at a finite proper time. Such geodesics are deemed incomplete. Furthermore, the celebrated Hawking-Penrose singularity theorems prove beyond doubt that under normal assumptions, all spacetime solutions of GR will have incomplete geodesics, and will therefore be singular [1-3].

It may be mentioned that the proof of the singularity theorems crucially depend on the fact that there exist congruences or a collection of nearby geodesics, such that they 
focus to the conjugate points in the past as well as in the future at finite proper times. This implies that the geodesics are no longer maximal curves in a pseudo-Riemannian manifold, contrary to their very definition as solutions of the geodesic equation. The only resolution of this apparent contradiction is to conclude that such geodesics are incomplete. The existence of conjugate points is a straightforward prediction of the Raychaudhuri equation [3].

In view of the above, in this article, we examine the issue of singularity resolution via the LQG modified Raychaudhuri equation, and in particular for the Schwarzschild solution in GR. Since the classical singularity is at the origin of the above black hole metric, $r=0$, we focus on the region inside the classical horizon at $r=2 G M$, where $M$ is the mass of the black hole and $G$ is Newton's constant (we work in $c=1=\hbar$ units). By choosing the appropriate regularized tetrads and holonomies, which are the conjugate variables in LQG, computing the corresponding expansion of geodesics and substituting in the Raychaudhuri equation, we show that they include effective repulsive terms which prevents the formation of conjugate points. This implies that the classical singularity theorems are rendered invalid and the singularity is resolved, at least for the spacetime under consideration. While our results strictly pertain to the static Schwarzschild spacetime, the robustness of our results indicate that the resolution will continue to hold for more complicated spacetimes, including those with little or no symmetries, and when quantum corrections from other sources are taken into account (e.g., in Refs. [4-6]).

As is well known, LQG [7] is one of the main nonperturbative approaches to the quantization of gravity. Within LQG, there have been numerous studies of both the interior and the full spacetime of black holes in four and lower dimensions [8-54]. These attempts were originally inspired by loop quantum cosmology (LQC), more precisely a certain quantization of the isotropic Friedmann-Lemaitre-Robertson-Walker (FLRW) model $[55,56]$ which uses a certain type of quantization of the phase space called polymer quantization [57-61]. This quantiztion introduces a parameter into the theory called the polymer scale that sets the minimal scale of the model. Close to this scale quantum effects become important. The approach in which such a parameter is taken to be constant is called the $\mu_{0}$ scheme (which in this paper we refer to as the $\stackrel{\mu}{\mu}$ scheme), while approaches where it depends on the phase space variables are denoted by $\bar{\mu}$ schemes. These various approaches were introduced to deal with some important issues resulting from quantization, namely, to have the correct classical limit (particularly in LQC), to avoid large quantum corrections near the horizon, and to have final physical results that are independent of auxiliary or fiducial parameters. Other approaches to this model in LQG such as Refs. [62-64] provide a derivation of a Schwarzschild black hole modified dynamics for the interior and the exterior regions, not relying on 
minisuperspace models. Starting from the full LQG theory, this model performs the symmetry reduction at the quantum level. This has led to several differences in the effective dynamics with respect to previous polymer quantization-inspired models, one of which is the absence of the formation of a white hole in the extended spacetime region replacing the classical singularity. All of these past studies in LQG and some other approaches (see, e.g., Refs. [65-67]) point to the resolution of the singularity at the effective level.

In this paper, we consider the interior of the Schwarzschild black hole expressed in terms of connection variables and follow the same polymer quantization as previous works based on minisuperspace models but study the behavior of modified effective geodesics in the interior of the black hole using the modified Raychaudhuri equation. We will consider both the $\stackrel{\leftrightarrow}{\mu}$ scheme and two of the most common cases in $\bar{\mu}$ schemes.

This paper is organized as follows. In Sec. 2, we review the classical interior of the Schwarzschild black hole. In Sec. 3, we remind the reader of the classical dynamics of the interior, derive the corresponding Raychaudhuri equation, and show that this leads to the expected presence of a singularity at the center of the black hole. In Sec. 4, we present the effective dynamics of the interior after polymer quantization in a general setting. We then go on to derive the effective Raychaudhuri equation of the $\stackrel{\mu}{\mu}$ scheme and two of the most common $\bar{\mu}$ schemes in Secs. 4.1, 4.2, and 4.3, respectively, showing

how the modified behavior of geodesics shows the resolution of singularity in each of these cases.

\section{Interior of the Schwarzschild black hole}

The celebrated metric of the exterior of a Schwarzschild black hole of mass $M$ is given by

$$
d s^{2}=-\left(1-\frac{2 G M}{r}\right) d t^{2}+\left(1-\frac{2 G M}{r}\right)^{-1} d r^{2}+r^{2}\left(d \theta^{2}+\sin ^{2} \theta d \phi^{2}\right)
$$

where and $r \in(0, \infty)$ is the radial coordinate distance and the radius of the 2 -spheres in Schwarzschild coordinates $(t, r, \theta, \phi)$. It is well known that for such a black hole, the timelike and spacelike curves switch their causal nature upon crossing the event horizon located at $R_{s}=2 G M$. Thus, the interior metric can be written as

$$
d s^{2}=-\left(\frac{2 G M}{t}-1\right)^{-1} d t^{2}+\left(\frac{2 G M}{t}-1\right) d r^{2}+t^{2}\left(d \theta^{2}+\sin ^{2} \theta d \phi^{2}\right) .
$$


Here and throughout the paper, $t$ is the Schwarzschild time with the range $t \in(0,2 G M)$. This metric is a special case of a Kantowski-Sachs cosmological spacetime that is given by the metric [68]

$$
\begin{aligned}
d s_{K S}^{2} & =-N(T)^{2} d T^{2}+g_{x x}(T) d x^{2}+g_{\theta \theta}(T) d \theta^{2}+g_{\phi \phi}(T) d \phi^{2} \\
& =-d \tau^{2}+g_{x x}(\tau) d x^{2}+g_{\Omega \Omega}(\tau) d \Omega^{2} .
\end{aligned}
$$

Note that $x$ here is not necessarily the radius $r$ of the 2 -spheres with area $A=4 \pi r^{2}$, where $N(T)$ is the lapse function corresponding to a generic time $T$. It is seen that the metric (2.2) and (2.3) are related by a transformation,

$$
d \tau^{2}=N(T)^{2} d T^{2}=\left(\frac{2 G M}{t}-1\right)^{-1} d t^{2} .
$$

The metric (2.3) represents a spacetime with spatial homogeneous but anisotropic foliations. A quick way to see this is that $g_{x x}(\tau)$ and $g_{\Omega \Omega}(\tau)$ can be considered as two distinct scale factors that affect the radial and angular parts of the metric separately. As is evident from (2.3), such a system is a minisuperspace model due to incorporating a finite number of configuration variables. Furthermore, it can be seen that the spatial hypersurfaces have topology $\mathbb{R} \times \mathbb{S}^{2}$, and the spatial symmetry group is the KantowskiSachs isometry group $\mathbb{R} \times S O(3)$. Due to this topology with a noncompact direction, $x \in \mathbb{R}$ in space, the symplectic form $\int_{\mathbb{R} \times \mathbb{S}^{2}} \mathrm{~d}^{3} x \mathrm{~d} q \wedge \mathrm{d} p$ diverges. Therefore, one needs to choose a finite fiducial volume over which this integral is calculated [9]. This is a common practice in the study of homogeneous minisuperspace models. Here one introduces an auxiliary length $L_{0}$ to restrict the noncompact direction to an interval $x \in \mathcal{I}=\left[0, L_{0}\right]$. The volume of the fiducial cylindrical cell in this case is $V_{0}=a_{0} L_{0}$, where $a_{0}$ is the area of the 2 -sphere $\mathbb{S}^{2}$ in $\mathcal{I} \times \mathbb{S}^{2}$.

In order to obtain the Hamiltonian of this system in connection variables, one first considers the full Hamiltonian of gravity written in terms of (the curvature) of the $s u(2)$ Ashtekar-Barbero connection $A_{a}^{i}$ and its conjugate momentum, the densitized triad $\tilde{E}_{a}^{i}$. Using the Kantowski-Sachs symmetry, these variables can be written as [9]

$$
\begin{aligned}
A_{a}^{i} \tau_{i} d x^{a} & =\frac{c}{L_{0}} \tau_{3} d x+b \tau_{2} d \theta-b \tau_{1} \sin \theta d \phi+\tau_{3} \cos \theta d \phi, \\
\tilde{E}_{i}^{a} \tau_{i} \partial_{a} & =p_{c} \tau_{3} \sin \theta \partial_{x}+\frac{p_{b}}{L_{0}} \tau_{2} \sin \theta \partial_{\theta}-\frac{p_{b}}{L_{0}} \tau_{1} \partial_{\phi},
\end{aligned}
$$

where $b, c, p_{b}$, and $p_{c}$ are functions that only depend on time and $\tau_{i}=-i \sigma_{i} / 2$ are a $s u(2)$ basis satisfying $\left[\tau_{i}, \tau_{j}\right]=\epsilon_{i j}{ }^{k} \tau_{k}$, with $\sigma_{i}$ being the Pauli matrices. Substituting these into the full Hamiltonian of gravity written in Ashtekar connection variables, one 
obtains the symmetry reduced Hamiltonian constraint adapted to this model as [9]

$$
H=-\frac{N}{2 G \gamma^{2}}\left[2 b c \sqrt{p_{c}}+\left(b^{2}+\gamma^{2}\right) \frac{p_{b}}{\sqrt{p_{c}}}\right],
$$

while the diffeomorphism constraint vanishes identically due to the homogenous nature of the model. Here, $\gamma$ is the Barbero-Immirzi parameter [7], and $p_{c} \geq 0$. $\gamma$ is the term whose inverse couples the first order Palatini action to a topological Nieh-Yan term yielding the Holst action. Hence it does not affect the equations of motion and classically has no effect on the system. However, after quantization, this parameter sets the size of the quantum of area in Planck units.

Using symmetry of the model, the full symplectic form

$$
\boldsymbol{\Omega}=\frac{1}{8 \pi G \gamma} \int_{\mathcal{I} \times \mathbb{S}^{2}} d^{3} x d A_{a}^{i}(\mathbf{x}) \wedge d \tilde{E}_{i}^{a}(\mathbf{y})
$$

reduces to $[9]$

$$
\boldsymbol{\Omega}=\frac{1}{2 G \gamma}\left(d c \wedge d p_{c}+2 d b \wedge d p_{b}\right)
$$

and consequently the Poisson brackets

$$
\left\{A_{a}^{i}(\mathbf{x}), \tilde{E}_{j}^{b}(\mathbf{y})\right\}=8 \pi G \gamma \delta_{j}^{i} \delta_{a}^{b} \delta^{3}(\mathbf{x}-\mathbf{y})
$$

reduce to

$$
\left\{c, p_{c}\right\}=2 G \gamma, \quad\left\{b, p_{b}\right\}=G \gamma .
$$

Furthermore, by substituting (2.5) and (2.6), and the components of the inverse of the metric (2.3), into the relation between the inverse triad and the spatial metric $q_{a b}$,

$$
q q^{a b}=\delta^{i j} \tilde{E}_{i}^{a} \tilde{E}_{j}^{b}
$$

one obtains for the generic metric (2.3) adapted to (2.5) and (2.6)

$$
\begin{aligned}
& g_{x x}(T)=\frac{p_{b}(T)^{2}}{L_{0}^{2} p_{c}(T)}, \\
& g_{\theta \theta}(T)=\frac{g_{\phi \phi}(T)}{\sin ^{2}(\theta)}=g_{\Omega \Omega}(T)=p_{c}(T) .
\end{aligned}
$$

Note that the lapse $N(T)$ is not determined and can be chosen as suited for a specific situation. Hence, the adapted metric using (2.13) and (2.14) becomes

$$
d s^{2}=-N(T)^{2} d T^{2}+\frac{p_{b}^{2}}{L_{0}^{2} p_{c}} d x^{2}+p_{c}\left(d \theta^{2}+\sin ^{2} \theta d \phi^{2}\right) .
$$


Comparing this metric written in Schwarzschild coordinates and lapse $N(t)$ with the standard Schwarzschild interior metric but with rescaled $r \rightarrow l x$,

$$
d s^{2}=-\left(\frac{2 G M}{t}-1\right)^{-1} d t^{2}+l^{2}\left(\frac{2 G M}{t}-1\right) d x^{2}+t^{2}\left(d \theta^{2}+\sin ^{2} \theta d \phi^{2}\right),
$$

we see that

$$
\begin{aligned}
N(t) & =\left(\frac{2 G M}{t}-1\right)^{-\frac{1}{2}}, \\
g_{x x}(t) & =\frac{p_{b}(t)^{2}}{L_{0}^{2} p_{c}(t)}=l^{2}\left(\frac{2 G M}{t}-1\right), \\
g_{\theta \theta}(T) & =\frac{g_{\phi \phi}(T)}{\sin ^{2}(\theta)}=g_{\Omega \Omega}(T)=p_{c}(t)=t^{2} .
\end{aligned}
$$

Hereafter we use $l=1$. This shows that

$$
\begin{array}{lll}
p_{b}=0, & p_{c}=4 G^{2} M^{2}, & \text { on the horizon } t=2 G M, \\
p_{b} \rightarrow 0, & p_{c} \rightarrow 0, & \text { at the singularity } t \rightarrow 0 .
\end{array}
$$

Also, note that in the fiducial volume, we can consider three surfaces $S_{x, \theta}, S_{x, \phi}$, and $S_{\theta, \phi}$, respectively, bounded by $\mathcal{I}$ and a great circle along a longitude of $V_{0}, \mathcal{I}$ and the equator of $V_{0}$, and the equator and a longitude with areas [9]

$$
\begin{aligned}
A_{x, \theta}=A_{x, \phi} & =2 \pi L_{0} \sqrt{g_{x x} g_{\Omega \Omega}}=2 \pi p_{b} \\
A_{\theta, \phi} & =\pi g_{\Omega \Omega}=\pi p_{c}
\end{aligned}
$$

with the volume of the fiducial region $\mathcal{I} \times \mathbb{S}^{2}$ given by [9]

$$
V=\int \mathrm{d}^{3} x \sqrt{|\operatorname{det} \tilde{E}|}=4 \pi L \sqrt{g_{x x}} g_{\Omega \Omega}=4 \pi p_{b} \sqrt{p_{c}}
$$

where $\sqrt{\operatorname{det}|\tilde{E}|}=\sqrt{q}$ with $q$ being the determinant of the spatial metric.

\section{Classical dynamics}

\subsection{Classical Hamiltonian and equations of motion}

We are interested in the classical dynamics of the interior of the Schwarzschild black hole in Ashtekar-Barbero connection formulation. As usual in gravity, the classical Hamiltonian is the sum of constraints that generate spacetime diffeomorphisms and 
internal or Gauss (in our case $s u(2)$ ) symmetry. The full version of the classical Hamiltonian constraint in this formulation is [7]

$$
H_{\text {full }}=\frac{1}{8 \pi G} \int d^{3} x \frac{N}{\sqrt{\operatorname{det}|\tilde{E}|}}\left\{\epsilon_{i}^{j k} F_{a b}^{i} \tilde{E}_{j}^{a} \tilde{E}_{k}^{b}-2\left(1+\gamma^{2}\right) K_{[a}{ }^{i} K_{b]}^{j} \tilde{E}_{i}^{a} \tilde{E}_{j}^{b}\right\},
$$

where $K_{a}^{i}$ is the extrinsic curvature of foliations and $\epsilon_{i j k}$ is the totally antisymmetric Levi-Civita symbol. Also, $F=d A+A \wedge A$ is the curvature of the Ashtekar-Barbero connection. The symmetry reduced Hamiltonian corresponding to the above full Hamiltonian is derived by substituting (2.5) and (2.6) in (3.1). In this way, one obtains $[9,11,13,29,52]$

$$
H=-\frac{N}{2 G \gamma^{2}}\left[\left(b^{2}+\gamma^{2}\right) \frac{p_{b}}{\sqrt{p_{c}}}+2 b c \sqrt{p_{c}}\right] .
$$

Given the homogeneous nature of the model, the diffeomorphism constraint is trivially satisfied, and after imposing the Gauss constraint, one is left only with the classical Hamiltonian constraint (3.2). In order to facilitate the derivation of the solutions to the equations of motion, we choose a gauge where the lapse function is

$$
N(T)=\frac{\gamma \sqrt{p_{c}(T)}}{b(T)}
$$

for which the Hamiltonian constraint becomes

$$
H=-\frac{1}{2 G \gamma}\left[\left(b^{2}+\gamma^{2}\right) \frac{p_{b}}{b}+2 c p_{c}\right] .
$$

The advantage of this lapse function is that the equations of motion of $c, p_{c}$ decouple from those of $b, p_{b}$,

$$
\begin{aligned}
& \frac{d b}{d T}=\{b, H\}=-\frac{1}{2}\left(b+\frac{\gamma^{2}}{b}\right), \\
& \frac{d p_{b}}{d T}=\left\{p_{b}, H\right\}=\frac{p_{b}}{2}\left(1-\frac{\gamma^{2}}{b^{2}}\right), \\
& \frac{d c}{d T}=\{c, H\}=-2 c \\
& \frac{d p_{c}}{d T}=\left\{p_{c}, H\right\}=2 p_{c} .
\end{aligned}
$$

These equations are also to be supplemented with the on-shell condition of the vanishing of the Hamiltonian constraint (3.4) on the constraint surface

$$
\left(b^{2}+\gamma^{2}\right) \frac{p_{b}}{b}+2 c p_{c} \approx 0,
$$


where $\approx$ stands for weak equality, i.e., on the constraint surface.

It is clear from (2.19) that $p_{c}$ is the square of the radius of the infalling 2 -spheres. In order to better understand the role of $b, c$ we use the relation of the proper time $\tau$ and a generic time $T$ for the metric (2.3),

$$
d \tau^{2}=-N(T)^{2} d T^{2}
$$

and the form of the lapse function (3.3), to rewrite Eqs. (3.8) as

$$
b=\frac{\gamma}{2} \frac{1}{\sqrt{p_{c}}} \frac{d p_{c}}{d \tau}=\gamma \frac{d}{d \tau} \sqrt{g_{\Omega \Omega}}=\frac{\gamma}{\sqrt{\pi}} \frac{d}{d \tau} \sqrt{A_{\theta, \phi}},
$$

where the last two terms on the right-hand side were derived using (2.23). Hence, classically, $b$ is proportional to the rate of change of the square root of the physical area of $\mathbb{S}^{2}$.

To interpret the role of $c$, we use the same method for (3.8), and by using (3.9) to replace $\gamma \frac{p_{b}}{b}=-\frac{b p_{b}}{\gamma}-\frac{2 c p_{c}}{\gamma}$ in the resultant expression and then using (2.22) and (2.23), we find out

$$
c=\gamma \frac{d}{d \tau}\left(\frac{p_{b}}{\sqrt{p_{c}}}\right)=\gamma \frac{d}{d \tau}\left(L_{0} \sqrt{g_{x x}}\right) .
$$

Hence, classically, $c$ is proportional to the rate of change of the physical length of $\mathcal{I}$.

The solution to the classical equations of motion (3.5)-(3.8) are be found to be

$$
\begin{aligned}
b(T) & = \pm \sqrt{e^{2 C_{1}} e^{-T}-\gamma^{2}}, \\
p_{b}(T) & =C_{2} e^{\frac{T}{2}} \sqrt{e^{2 C_{1}}-\gamma^{2} e^{T}}, \\
c(T) & =C_{3} e^{-2 T}, \\
p_{c}(T) & =C_{4} e^{2 T} .
\end{aligned}
$$

Since we know from (2.19) that in Schwarzschild coordinates $p_{c}(t)=t^{2}$, and considering the fourth equation above, we see that a transformation $T=\ln (t)$ can lead to such a solution for $p_{c}$. Under such a transformation, the above equations become

$$
\begin{aligned}
b(t) & = \pm \sqrt{\frac{e^{2 C_{1}}}{t}-\gamma^{2}}, \\
p_{b}(t) & =C_{2} t \sqrt{\frac{e^{2 C_{1}}}{t}-\gamma^{2}}, \\
c(t) & =\frac{C_{3}}{t^{2}}, \\
p_{c}(t) & =C_{4} t^{2} .
\end{aligned}
$$


Considering (2.19), we see that

$$
C_{4}=1
$$

Also, from (2.20), we can deduce

$$
0=p_{b}(2 G M)=2 G M C_{2} \sqrt{\frac{e^{2 C_{1}}}{2 G M}-\gamma^{2}},
$$

which yields

$$
C_{1}=\frac{1}{2} \ln \left(2 G M \gamma^{2}\right)
$$

Next, from (2.18), we see

$$
p_{b}(t)^{2}=l^{2}\left(\frac{2 G M}{t}-1\right) L_{0}^{2} t^{2},
$$

which if compared with (3.18) and using (3.23) yields

$$
C_{2}=\frac{l L_{0}}{\gamma}
$$

Finally, using (3.9), we get

$$
C_{3}=\mp \gamma G M l L_{0} .
$$

Hence, the equations of motion in Schwarzschild written in $t$ become

$$
\begin{aligned}
b(t) & = \pm \gamma \sqrt{\frac{2 G M}{t}-1}, \\
p_{b}(t) & =l L_{0} t \sqrt{\frac{2 G M}{t}-1}, \\
c(t) & =\mp \frac{\gamma G M l L_{0}}{t^{2}}, \\
p_{c}(t) & =t^{2} .
\end{aligned}
$$

The behavior of these solutions as a function of $t$ is depicted in Fig. 1. From these equations or the plot, one can see that $p_{c} \rightarrow 0$ as $t \rightarrow 0$. This means that at the classical singularity, the Riemann invariants such as the Kretschmann scalar

$$
K=R_{a b c d} R^{a b c d} \propto \frac{1}{p_{c}^{3}},
$$

all diverge, signaling the presence of a physical singularity there as expected.

We can also see from Fig. 1 that $b$, the rate of change of the square root of the physical area of $\mathbb{S}^{2}$, as well as $c$, the rate of change of the physical length of $\mathcal{I}$, diverge at the classical singularity. 


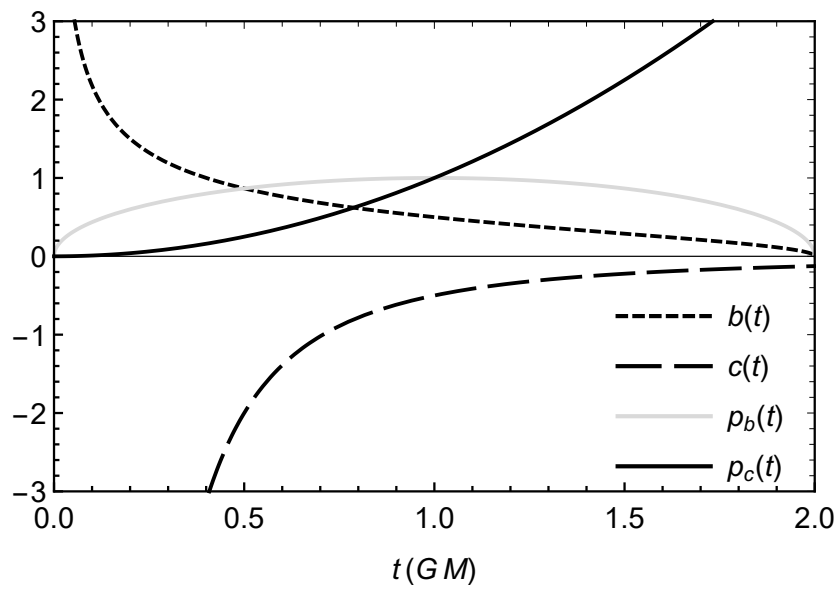

Figure 1. The behavior of canonical variables as a function of Schwarzschild time $t$. We have chosen the positive sign for $b$ and negative sign for $c$. The figure is plotted using $\gamma=0.5, M=$ $1, G=1$, and $L_{0}=1$.

\subsection{Classical Raychaudhuri equation}

The celebrated Raychaudhuri equation [3]

$$
\frac{d \theta}{d \tau}=-\frac{1}{3} \theta^{2}-\sigma_{a b} \sigma^{a b}+\omega_{a b} \omega^{a b}-R_{a b} U^{a} U^{b}
$$

describes the behavior of geodesics in spacetime purely geometrically and independent of the theory of gravity under consideration. Here, $\theta$ is the expansion term describing how geodesics focus or defocus; $\sigma_{a b} \sigma^{a b}$ is the shear which describes how, e.g., a circular configuration of geodesics changes shape into, say, an ellipse; $\omega_{a b} \omega^{a b}$ is the vorticity term; $R_{a b}$ is the Ricci tensor; and $U^{a}$ is the tangent vector to the geodesics. Note that, due the sign of the expansion, shear, and the Ricci term, they all contribute to focusing, while the vorticity terms leads to defocusing.

In our case, since we consider the model in vacuum, $R_{a b}=0$. Also, in general in Kantowski-Scahs models, the vorticity term is only nonvanishing if one considers metric perturbations [68]. Hence, $\omega_{a b} \omega^{a b}=0$ in our model, too. This reduces the Raychaudhuri equation for our analysis to

$$
\frac{d \theta}{d \tau}=-\frac{1}{3} \theta^{2}-\sigma_{a b} \sigma^{a b}
$$

One can show that the above implies the convergence of geodesics to conjugate points at a finite proper time $\tau_{0}<3 /\left|\theta_{0}\right|$, where $\theta_{0}$ is the starting expansion [3]. This is due to the fact that the right-hand side of the above is negative, which in turn is a direct 
consequence of the universal and attractive nature of gravity. This also reiterates the "inevitability" of geodesics focusing and the consequent singularity theorems.

In order to adapt the Raychaudhuri equation to the current LQG formalism, we write the quantities $\theta$ and $\sigma^{2}=\sigma_{a b} \sigma^{a b}$ appearing on the right-hand side of (3.33) in terms of the canonical variables as [13]

$$
\begin{aligned}
\theta & =\frac{\dot{p}_{b}}{N p_{b}}+\frac{\dot{p}_{c}}{2 N p_{c}} \\
\sigma^{2} & =\frac{2}{3}\left(-\frac{\dot{p}_{b}}{N p_{b}}+\frac{\dot{p}_{c}}{N p_{c}}\right)^{2} .
\end{aligned}
$$

Replacing these in (3.33) and using the equations of motion (3.5)-(3.8) one obtains

$$
\frac{d \theta}{d \tau}=-\frac{1}{2 p_{c}}\left(1+\frac{9 b^{2}}{2 \gamma^{2}}+\frac{\gamma^{2}}{2 b^{2}}\right)
$$

As expected, the right hand side is negative ( since $\left.p_{c}>0\right)$ and diverges at the singularity given the behavior of canonical variables in Fig. 1. This can also be seen from the expression (3.36) written in terms of $t$, by using the solutions (3.27)-(3.30) and the lapse (2.17) in (3.36) to get

$$
\frac{d \theta}{d \tau}=\frac{-2 t^{2}+8 G M t-9 G^{2} M^{2}}{t^{\frac{5}{2}}(2 G M-t)^{\frac{3}{2}}} .
$$

The behavior of $\frac{d \theta}{d \tau}$ from (3.37) is presented in Fig. (2). This figure confirms that $\frac{d \theta}{d \tau}$ diverges at the classical singularity, pointing to an infinite focusing of geodesics at that region. All of these observations are well known. In what follows, we show that the quantum effects modify this behavior particularly close to the classical singularity.

\section{Effective dynamics and Raychaudhuri equation}

The effective behavior of the interior of the Schwarzschild black hole can be deduced from its effective Hamiltonian (constraint). There are various ways to obtain such an effective Hamiltonian from the classical one. Usually one first obtains the quantum Hamiltonian constraint. This is done by first writing the full Hamiltonian (3.1), particularly the curvature term(s), in terms of holonomies $h_{x}, h_{\theta}, h_{\phi}$ along edges in the radial and angular direction, and fluxes, instead of the connection and the triad $[9,13,29,69]$. The main reason to do so is that in loop quantum gravity, the connection is not a well-defined operator on the Hilbert space of the theory [7].

One then represents the holonomies and fluxes, and thus the Hamiltonian constraint as an operator on a suitable Hilbert space [7]. Note that, due to the nonexistence of 


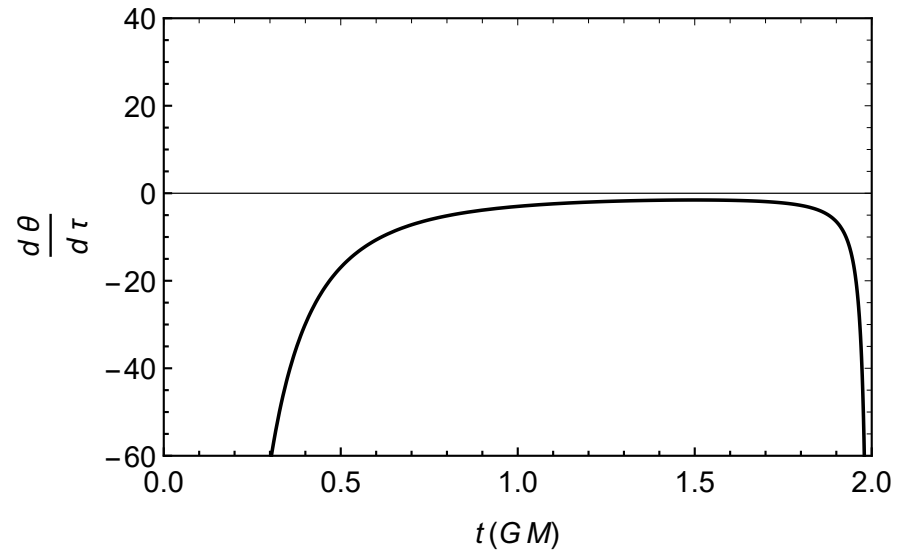

Figure 2. The right-hand side of the Raychaudhuri equation as a function of Schwarzschild time $t$. At both the classical singularity $t \rightarrow 0$ and at the horizon $t=2 G M, \frac{d \theta}{d \tau}$ diverges. The former is due to a physical singularity while the latter happens because of the choice of coordinate system. To draw this plot, we have set $M=1, G=1$.

connection on the Hilbert space, such a representation is unitarily inequivalent to the usual Schrodinger representation [7]. As a consequence, one obtains distinct physical results compared to the Schrodinger representation. Another property of LQG representation is that there exist no infinitesimal diffeomorphisms, and one only has access to finite diffeomorphisms. This leads to the discretization of space. For finite dimensional systems, such a representation is isomorphic to the polymer representation. This is a representation in which some of the operators are not weakly continuous in their parameters [58-61]. In this case, the unitary inequivalence to the Schrodiner representation follows directly from the Stone-von Neumann theorem [70]. Due to the existence of only finite transformations generated by some operators, minimal scales appear in the theory, which then leads to the discretization of some elements of the theory depending on what operators exhibit only finite transformations. Usually, these minimal scales are denoted by $\mu$ as we will see below.

After obtaining the quantum Hamiltonian as mentioned above, one finds an effective Hamiltonian by either using a path integral approach, or by acting the quantum Hamiltonian on states peaked around some classical solutions [52, 57-61, 71]. These methods will lead to an effective Hamiltonian that can also be heuristically obtained by replacing

$$
\begin{aligned}
& b \rightarrow \frac{\sin \left(\mu_{b} b\right)}{\mu_{b}}, \\
& c \rightarrow \frac{\sin \left(\mu_{c} c\right)}{\mu_{c}}
\end{aligned}
$$


in the classical Hamiltonian.

The free parameters $\mu_{b}, \mu_{c}$ are the minimum scales associated with the radial and angular directions $[9,13,29,69]$. In LQG, there exist two general schemes regarding these $\mu$ parameters. In one, called the $\mu_{0}$ scheme, $\mu$ parameters are considered to be constant $[9,42,48,72]$. Applying such a scheme to isotropic and Bianchi-I cosmological models, however, has shown to lead to incorrect semiclassical limit. To remedy this and other issues regarding the appearance of large quantum effects at the horizon or dependence of physical quantities on fiducial variables, new schemes referred to as the $\bar{\mu}$ scheme or "improved dynamics" have been proposed in which $\mu$ parameters depend on canonical variables $[11,29,69,73]$. This scheme is itself divided into various different ways of expressing the dependence of $\mu$ parameters on canonical variables. In addition, new $\mu_{0}$ schemes have also been put forward (e.g., Refs. [13, 44]) with the intent of resolving the aforementioned issues.

In case of the Schwarzschild interior due to lack of matter content, it is not clear which scheme does not lead to the correct semiclassical limit. Hence for completeness, in this paper, we will study the modifications to the Raychaudhuri equation in the constant $\mu$ scheme, which here we call the $\stackrel{\circ}{\mu}$ scheme, as well as in two of the most common improved schemes, which we denote by $\bar{\mu}$ and $\bar{\mu}^{\prime}$ schemes.

Applying any of the methods of deriving an effective Hamiltonian or simply replacing (4.1) and (4.2) into the classical Hamiltonian (3.2), one obtains an effective Hamiltonian constraint,

$$
H_{\mathrm{eff}}^{(N)}=-\frac{N}{2 G \gamma^{2}}\left[\left(\frac{\sin ^{2}\left(\mu_{b} b\right)}{\mu_{b}^{2}}+\gamma^{2}\right) \frac{p_{b}}{\sqrt{p_{c}}}+2 \frac{\sin \left(\mu_{b} b\right)}{\mu_{b}} \frac{\sin \left(\mu_{c} c\right)}{\mu_{c}} \sqrt{p_{c}}\right] .
$$

In order to be able to find the deviations from the classical behavior, we need to use the same lapse as we did in the classical part. Under (4.1), this lapse (3.3) becomes

$$
N=\frac{\gamma \mu_{b} \sqrt{p_{c}}}{\sin \left(\mu_{b} b\right)}
$$

Using this in (4.3) yields

$$
H_{\text {eff }}=-\frac{1}{2 \gamma G}\left[p_{b}\left[\frac{\sin \left(\mu_{b} b\right)}{\mu_{b}}+\gamma^{2} \frac{\mu_{b}}{\sin \left(\mu_{b} b\right)}\right]+2 p_{c} \frac{\sin \left(\mu_{c} c\right)}{\mu_{c}}\right] .
$$

Note that both (4.3) and (4.5) reduce to their classical counterparts (3.2) and (3.4) respectively, as is expected. 


\section{$4.1 \quad$ $\mu$ scheme}

As mentioned before, in this scheme, one assumes that the polymer or minimal scales $\stackrel{\circ}{\mu}_{b}, \stackrel{\circ}{\mu}_{c}$ are constants. Hence, the equations of motion corresponding to (4.5) become

$$
\begin{aligned}
& \frac{d b}{d T}=\left\{b, H_{\mathrm{eff}}\right\}=-\frac{1}{2}\left[\frac{\sin \left(\stackrel{\circ}{\mu}_{b} b\right)}{\stackrel{\circ}{\mu}_{b}}+\gamma^{2} \frac{\stackrel{\circ}{\mu}_{b}}{\sin \left(\stackrel{\circ}{\mu}_{b} b\right)}\right], \\
& \frac{d p_{b}}{d T}=\left\{p_{b}, H_{\mathrm{eff}}\right\}=\frac{1}{2} p_{b} \cos \left(\stackrel{\circ}{\mu} b_{b} b\right)\left[1-\gamma^{2} \frac{\stackrel{\circ}{\mu}_{b}^{2}}{\sin ^{2}\left(\stackrel{\circ}{\mu}_{b} b\right)}\right] \text {, } \\
& \frac{d c}{d T}=\left\{c, H_{\mathrm{eff}}\right\}=-2 \frac{\sin \left(\stackrel{\circ}{\mu}_{c} c\right)}{\stackrel{\circ}{\mu}_{c}}, \\
& \frac{d p_{c}}{d T}=\left\{p_{c}, H_{\mathrm{eff}}\right\}=2 p_{c} \cos \left(\dot{\mu}_{c} c\right) .
\end{aligned}
$$

Notice that the $\stackrel{\circ}{\mu}_{b} \rightarrow 0$ and $\stackrel{\circ}{\mu}_{c} \rightarrow 0$ limit of these equations corresponds to the classical equations of motion (3.5)-(3.8). The solutions to these equations in terms of the Schwarzschild time $t$ (after a transformation $T=\ln (t)$ ) and choosing suitable initial conditions, are given by

$$
\begin{aligned}
& b(t)=\frac{\cos ^{-1}\left[\sqrt{1+\gamma^{2} \stackrel{\circ}{\mu}_{b}^{2}} \tanh \left(\sqrt{1+\gamma^{2} \stackrel{\circ}{\mu}_{b}^{2}} \ln \left[\frac{2 \sqrt{\frac{t}{2 M}}}{\gamma \mu_{b}}\right]\right)\right]}{\stackrel{\circ}{h}_{b}}, \\
& p_{b}(t)=\frac{\gamma \stackrel{\mu}{\mu}_{b} L_{0} M\left(\frac{\gamma^{2} \stackrel{\leftrightarrow}{\mu}_{c}^{2} L_{0}^{2} M^{2}}{4 t^{2}}+t^{2}\right) \sqrt{1-\left(1+\gamma^{2} \ddot{\mu}_{b}^{2}\right) \tanh ^{2}\left(\sqrt{\gamma^{2} \grave{\mu}_{b}^{2}+1} \ln \left[\frac{2 \sqrt{\frac{t}{2 M}}}{\gamma \dot{\mu}_{b}}\right]\right)}}{t^{2} \sqrt{\frac{\gamma^{2} \dot{\mu}_{c}^{2} L_{0}^{2} M^{2}}{4 t^{4}}+1}\left(\gamma^{2} \stackrel{\circ}{\mu}_{b}^{2}-\left(1+\gamma^{2} \dot{\mu}_{b}^{2}\right) \tanh ^{2}\left(\sqrt{1+\gamma^{2} \dot{\mu}_{b}^{2}} \ln \left[\frac{2 \sqrt{\frac{t}{2 M}}}{\gamma \dot{\mu}_{b}}\right]\right)+1\right)}, \\
& c(t)=-\frac{\tan ^{-1}\left(\frac{\gamma \check{\rho}_{c} L_{0} M}{2 t^{2}}\right)}{\check{\rho}_{c}}, \\
& p_{c}(t)=\frac{\gamma^{2} \ddot{\mu}_{c}^{2} L_{0}^{2} M^{2}}{4 t^{2}}+t^{2} .
\end{aligned}
$$

The behavior of these solutions is plotted in Fig. 3. It is seen that both $p_{b}$ and $p_{c}$ exhibit a bounce in this effective regime. Particularly, there is a minimum radius-atthe-bounce due to the existence of a minimum value for $p_{c}$. This leads to the resolution of the classical singularity which can, e.g., be seen from the fact that the Riemann invariants, which are proportional to $\frac{1}{p_{c}^{n}}$ with $n>0$, do not diverge anywhere inside the black hole. 


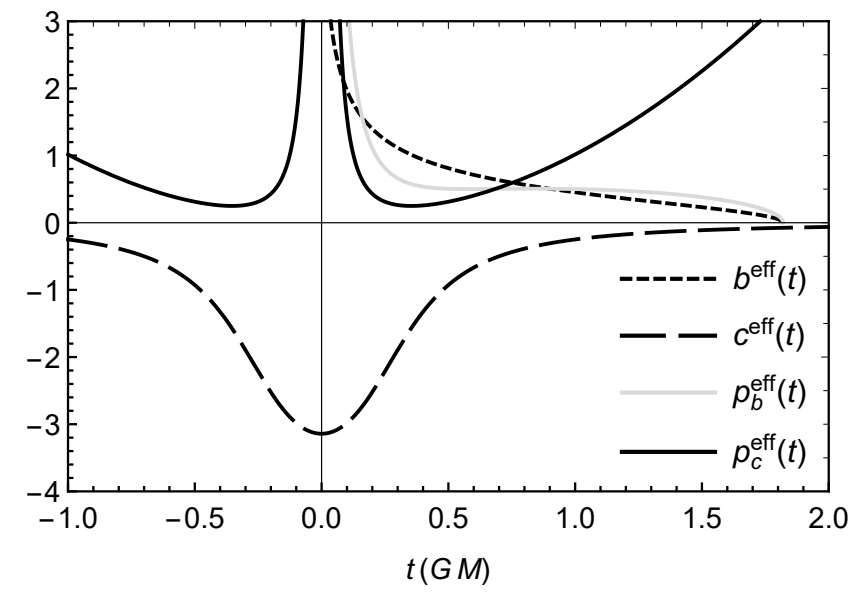

Figure 3. The behavior of canonical variables as a function of Schwarzschild time $t$. We have chosen the positive sign for $b$ and negative sign for $c$. The figure is plotted using $\gamma=0.5, M=$ $1, G=1, L_{0}=1$ and $\stackrel{\circ}{\mu}_{b}=0.5=\stackrel{\circ}{\mu}_{c}$. Notice the bounce in $p_{b}, p_{c}$ and also in $c$.

Replacing the effective solutions (4.7) and (4.9) into (3.34) and (3.35) and using them in the expression of the Raychaudhuri equation (3.33), one obtains

$$
\begin{aligned}
& \frac{d \theta}{d \tau}=\frac{1}{\gamma^{2} p_{c}} \frac{\sin ^{2}\left(\stackrel{\circ}{\mu}_{b} b\right)}{\check{\mu}_{b}^{2}}\left[\cos \left(\stackrel{\circ}{\mu}_{b} b\right) \cos \left(\stackrel{\circ}{\mu}_{c} c\right)-\frac{\cos ^{2}\left(\stackrel{\circ}{\mu}_{b} b\right)}{4}-3 \cos ^{2}\left(\stackrel{\circ}{\mu}_{c} c\right)\right] \\
& +\frac{\cos \left(\stackrel{\circ}{\mu}_{b} b\right)}{p_{c}}\left[\frac{\cos \left(\stackrel{\circ}{\mu} b_{b} b\right)}{2}-\cos \left(\stackrel{\circ}{\mu}_{c} c\right)-\frac{\gamma^{2}}{4} \cos \left(\stackrel{\circ}{\mu}_{b} b\right) \frac{\stackrel{\circ}{\mu}_{b}^{2}}{\sin ^{2}\left(\stackrel{\circ}{\mu}_{b} b\right)}\right] .
\end{aligned}
$$

Before considering the full nonperturbative expression above, let us look at its expansion up to the second order in $\stackrel{\circ}{\mu}_{b}$ and $\stackrel{\circ}{\mu}_{c}$,

$$
\frac{d \theta}{d \tau} \approx-\frac{1}{2 p_{c}}\left(1+\frac{9 b^{2}}{2 \gamma^{2}}+\frac{\gamma^{2}}{2 b^{2}}\right)+\stackrel{\circ}{\mu}_{b}^{2} \frac{1}{2 p_{c}}\left(\frac{b^{4}}{\gamma^{2}}+\frac{\gamma^{2}}{3}\right)+\stackrel{\circ}{\mu}_{c}^{2} \frac{c^{2}}{2 p_{c}}\left(1+\frac{5 b^{2}}{\gamma^{2}}\right) .
$$

One can see that the first term on the right-hand side is the classical expression (3.36) which is always negative and leads to the divergence of classical expansion rate at the singularity, i.e., infinite focusing. However, Eq. (4.15) now involves two additional effective terms proportional to $\stackrel{\mu}{b}_{b}^{2}$ and $\dot{\mu}_{c}^{2}$, both of which are positive. Hence, the quantum corrections up to the second order in polymer parameters contribute to defocusing, which becomes particularly large close to the singularity.

This is in fact confirmed by considering the full nonperturbative expression (4.14) written in terms of the Schwarzschild time $t$ using the solutions (4.10)-(4.13). We do not present this expression here since it is very lengthy. However, we have plotted this expression in Fig. 4. This plot includes the classical versus the effective behavior of $\frac{d \theta}{d \tau}$ 


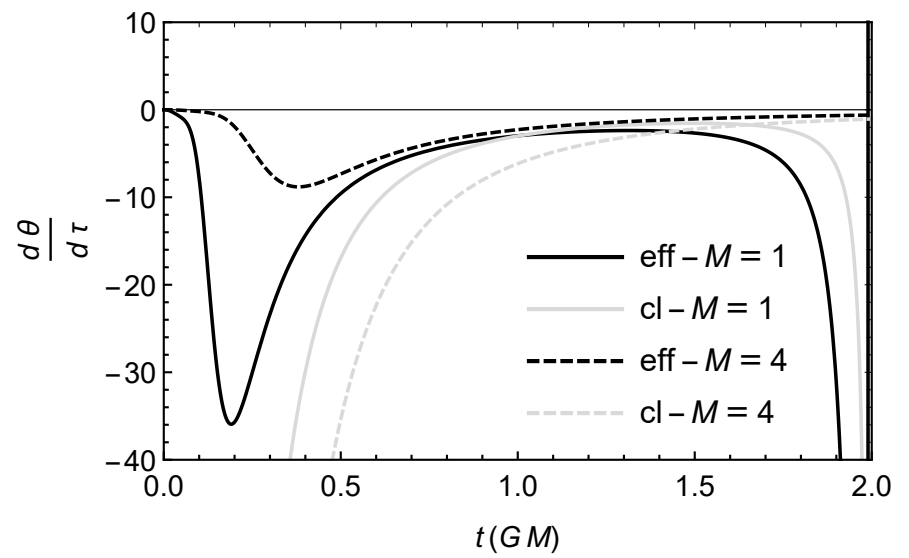

Figure 4. Plot of $\frac{d \theta}{d \tau}$ as a function of the Schwarzschild time $t$, for two different masses in classical vs effective regimes. The figure is plotted using $\gamma=0.5, G=1, L_{0}=1$, and $\stackrel{\circ}{\mu}_{b}=0.08=\stackrel{\circ}{\mu}_{c}$.
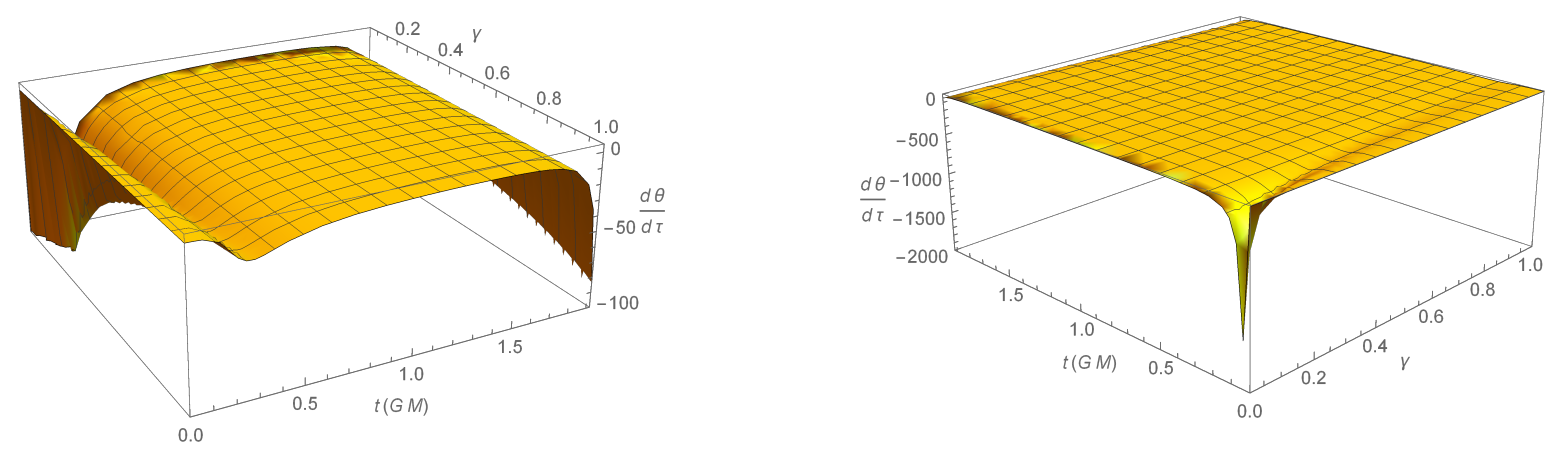

Figure 5. Left: plot of $\frac{d \theta}{d \tau}$ as a function of both $t$ and $\gamma$. Right: The same plot with the full interval of $\frac{d \theta}{d \tau}$ in picture. The values used here are $G=1, L_{0}=1$, and $\stackrel{\circ}{\mu}_{b}=0.08=\stackrel{\circ}{\mu}_{c}$.

for two different masses. First, we can see that, while $\frac{d \theta}{d \tau}$ diverges at the classical singularity, signaling an infinite focusing of geodesics there for both masses, the quantum effects actually reverse this situation for the effective case. Consequently, $\frac{d \theta}{d \tau}$ bounces back from a finite negative value and goes to zero when we approach the classical singularity region. This leads to the resolution of the singularity in the effective theory. Second, note that this happens earlier for a larger black hole.

Looking back at the Raychaudhuri equation (3.33), we see that both terms in that equation carry a negative sign, so, classically, they both contribute to focusing of geodesics, and this focusing becomes larger and larger with no other term being present to counter it. However, the nonperturbative effective correction terms resulting from loop quantization contribute to terms that have a positive sign and only become 

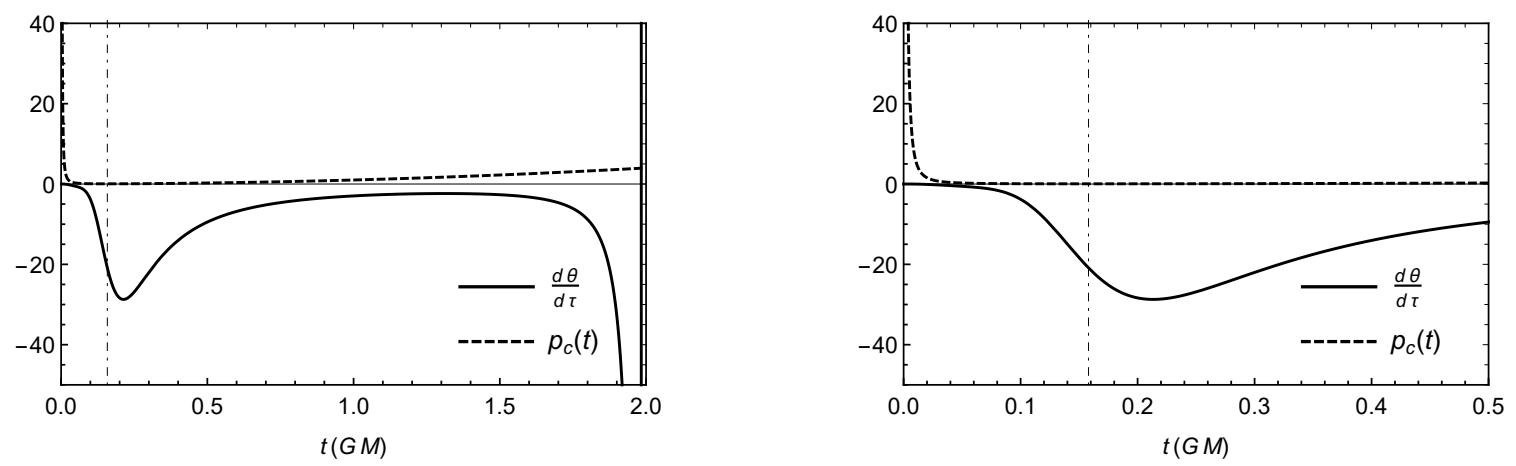

Figure 6. Left: plot of $\frac{d \theta}{d \tau}$ vs $p_{c}(t)$ as a function of the Schwarzschild time $t$. Right: the close-up of $0 \leq t \leq 0.5 G M$ portion of the left figure. The vertical dot-dashed line shows the time $t \approx 0.158 G M$ when the minimum of $p_{c}^{\min }=0.05$ happens in this case. These are plotted using $\gamma=0.5, G=1, L_{0}=1$, and $\stackrel{\circ}{\mu}_{b}=0.1=\stackrel{\circ}{\mu}_{c}$.

significant when one gets close to the singularity where quantum gravity effects should be significant. These effective terms then take over and revert the focusing property of the classical terms, so much so that they return the value of $\frac{d \theta}{d \tau}$ to zero at the region that used to be the classical singularity.

To get more insight, we also have plotted $\frac{d \theta}{d \tau}$ against the (square of the) radius of 2 -spheres, $p_{c}(t)$, in Fig. 6. From this figure, one can see that both $p_{c}$ and $\frac{d \theta}{d \tau}$ bounce back. However, $\frac{d \theta}{d \tau}$ starts bouncing back much earlier than $p_{c}$ and "knows" about the "repulsive" quantum gravity effects much more earlier than $p_{c}$ does. By the time $p_{c}$ starts bouncing back, $\frac{d \theta}{d \tau}$ is already bouncing toward zero value.

Finally, in Fig. 5, we have plotted $\frac{d \theta}{d \tau}$ as a function of both $t$ and the BarberoImmirzi parameter $\gamma$. It is seen that with given $\stackrel{\circ}{b}_{b}$ and $\stackrel{\circ}{\mu}_{c}$, decrease in value of $\gamma$ deepens the minimum of $\frac{d \theta}{d \tau}$ but in $\stackrel{\circ}{\mu}$ scheme there always will be a bounce anyway while $\gamma>0$.

\section{$4.2 \bar{\mu}$ scheme}

In this scheme $\bar{\mu}_{b}, \bar{\mu}_{c}$ are assumed to depend on the triad components as

$$
\begin{aligned}
& \bar{\mu}_{b}=\sqrt{\frac{\Delta}{p_{b}}}, \\
& \bar{\mu}_{c}=\sqrt{\frac{\Delta}{p_{c}}} .
\end{aligned}
$$




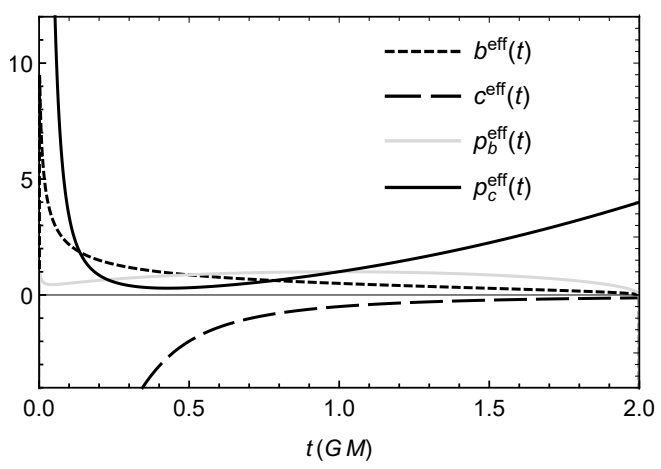

Figure 7. The behavior of canonical variables as a function of Schwarzschild time $t$ in the $\bar{\mu}$ scheme. We have chosen the positive sign for $b$ and negative sign for $c$. The figure is plotted using $\gamma=0.5, M=1, G=1, L_{0}=1$, and $\Delta=0.1$.

Using the same lapse as (4.4) but keeping in mind the above dependence of $\bar{\mu}_{b}$, $\bar{\mu}_{c}$, one can easily obtain the equations of motion as

$$
\begin{aligned}
\frac{d b}{d T} & =\frac{1}{4}\left(b \cos \left(\bar{\mu}_{b} b\right)-3 \frac{\sin \left(\bar{\mu}_{b} b\right)}{\bar{\mu}_{b}}-\gamma^{2} \frac{\bar{\mu}_{b}}{\sin \left(\bar{\mu}_{b} b\right)}\left[1+b \cos \left(\bar{\mu}_{b} b\right) \frac{\bar{\mu}_{b}}{\sin \left(\bar{\mu}_{b} b\right)}\right]\right), \\
\frac{d p_{b}}{d T} & =\frac{1}{2} p_{b} \cos \left(\bar{\mu}_{b} b\right)\left[1-\gamma^{2} \frac{\bar{\mu}_{b}^{2}}{\sin ^{2}\left(\bar{\mu}_{b} b\right)}\right], \\
\frac{d c}{d T} & =c \cos \left(\bar{\mu}_{c} c\right)-3 \frac{\sin \left(\bar{\mu}_{c} c\right)}{\bar{\mu}_{c}}, \\
\frac{d p_{c}}{d T} & =2 p_{c} \cos \left(\bar{\mu}_{c} c\right) .
\end{aligned}
$$

These solutions are plotted in Fig. 7. To obtain them we have solved the system numerically and used the same initial conditions very close to the horizon as the classical solutions. From Fig. 7 one can see that $p_{c}$ still retains a certain minimum value and bounces after reaching this value. Hence, again, none of the Riemann invariants will diverge.

The full Raychaudhuri equation now becomes

$$
\begin{aligned}
\frac{d \theta}{d \tau}= & \frac{1}{\gamma^{2} p_{c}} \frac{\sin ^{2}\left(\bar{\mu}_{b} b\right)}{\bar{\mu}_{b}^{2}}\left[\cos \left(\bar{\mu}_{b} b\right) \cos \left(\bar{\mu}_{c} c\right)-\frac{\cos ^{2}\left(\bar{\mu}_{b} b\right)}{4}-3 \cos ^{2}\left(\bar{\mu}_{c} c\right)\right] \\
& +\frac{\cos \left(\bar{\mu}_{b} b\right)}{p_{c}}\left[\frac{\cos \left(\bar{\mu}_{b} b\right)}{2}-\cos \left(\bar{\mu}_{c} c\right)-\frac{\gamma^{2}}{4} \cos \left(\bar{\mu}_{b} b\right) \frac{\bar{\mu}_{b}^{2}}{\sin ^{2}\left(\bar{\mu}_{b} b\right)}\right] .
\end{aligned}
$$

This looks similar in form to (4.14), but we should keep in mind that the polymer parameters here depend on canonical variables as in (4.16) and (4.17). As in the previous case, let us consider the perturbative expansion of this expression before considering 

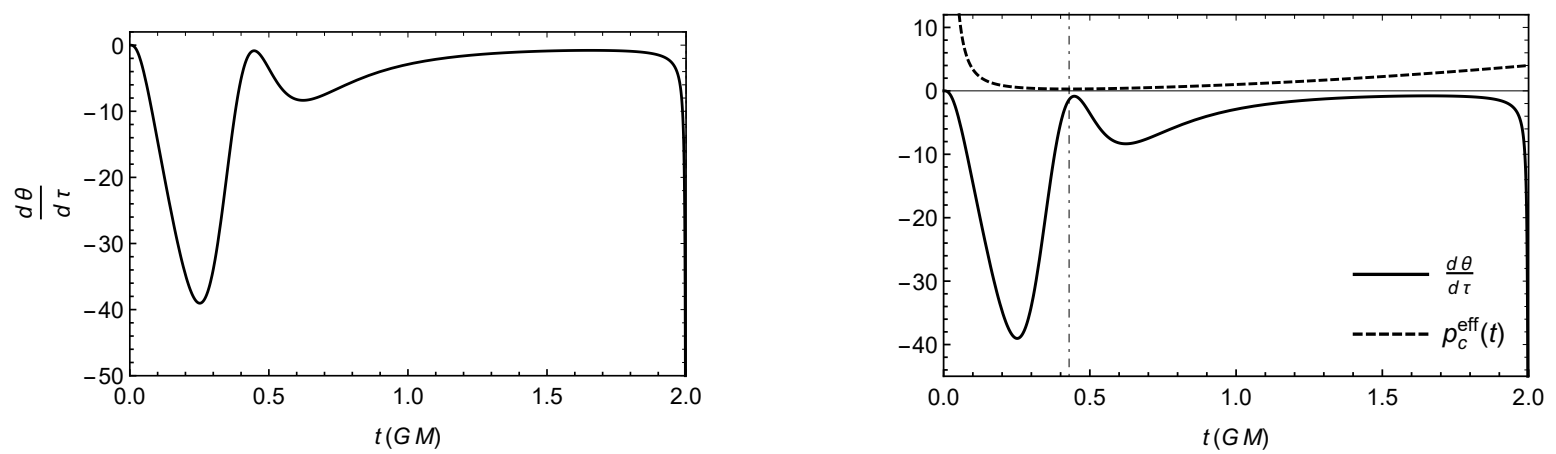

Figure 8. Left: Raychaudhuri equation in the $\bar{\mu}$ scheme. Right: Raychaudhuri equation vs $p_{c}$. The vertical dot-dashed line at $t \approx 0.43 G M$ is the position of the bounce of $p_{c}$ where its minimum $p_{c}^{\min }=0.29$ happens in this case. The figure is plotted using $\gamma=0.5, M=1, G=$ $1, L_{0}=1$, and $\Delta=0.1$.

the full nonperturbative version. Up to first order in $\Delta$ (which can be considered as the second order in $\bar{\mu}$ scales), we get

$$
\frac{d \theta}{d \tau} \approx-\frac{1}{2 p_{c}}\left(1+\frac{9 b^{2}}{2 \gamma^{2}}+\frac{\gamma^{2}}{2 b^{2}}\right)+\frac{\Delta}{p_{c}}\left[\frac{1}{6 p_{b}}\left(\frac{3 b^{4}}{\gamma^{2}}+\gamma^{2}\right)+\frac{c^{2}}{2 p_{c}}\left(1+\frac{5 b^{2}}{\gamma^{2}}\right)\right] .
$$

Once again, the first term on the right-hand side is the classical expression of the Raychaudhuri equation (3.36), which contributes to infinite focusing at the singularity. However, all the correction terms are positive and once again these terms contribute to defocusing which becomes significant close to the singularity.

The full nonperturbative form the modified Raychaudhuri equation in terms of $t$ is plotted in Fig. 8. We see that, approaching from the horizon to where the classical singularity used to be, an initial bump or bounce in encountered, followed by a more pronounced bounce closer to where the singularity used to be. Once again, the quantum corrections become dominant close to the singularity and turn back the $\frac{d \theta}{d \tau}$ such that at $t \rightarrow 0$ no focusing happens at all. Furthermore, from the right plot in Fig. 8, we see that the first bounce in the Raychaudhuri equation happens much earlier than the bounce in $p_{c}$.

\section{$4.3 \quad \bar{\mu}^{\prime}$ scheme}

Here $\bar{\mu}_{b}^{\prime}, \bar{\mu}_{c}^{\prime}$ have the following dependence on the triad components,

$$
\begin{aligned}
\bar{\mu}_{b}^{\prime} & =\sqrt{\frac{\Delta}{p_{c}}}, \\
\bar{\mu}_{c}^{\prime} & =\frac{\sqrt{p_{c} \Delta}}{p_{b}} .
\end{aligned}
$$



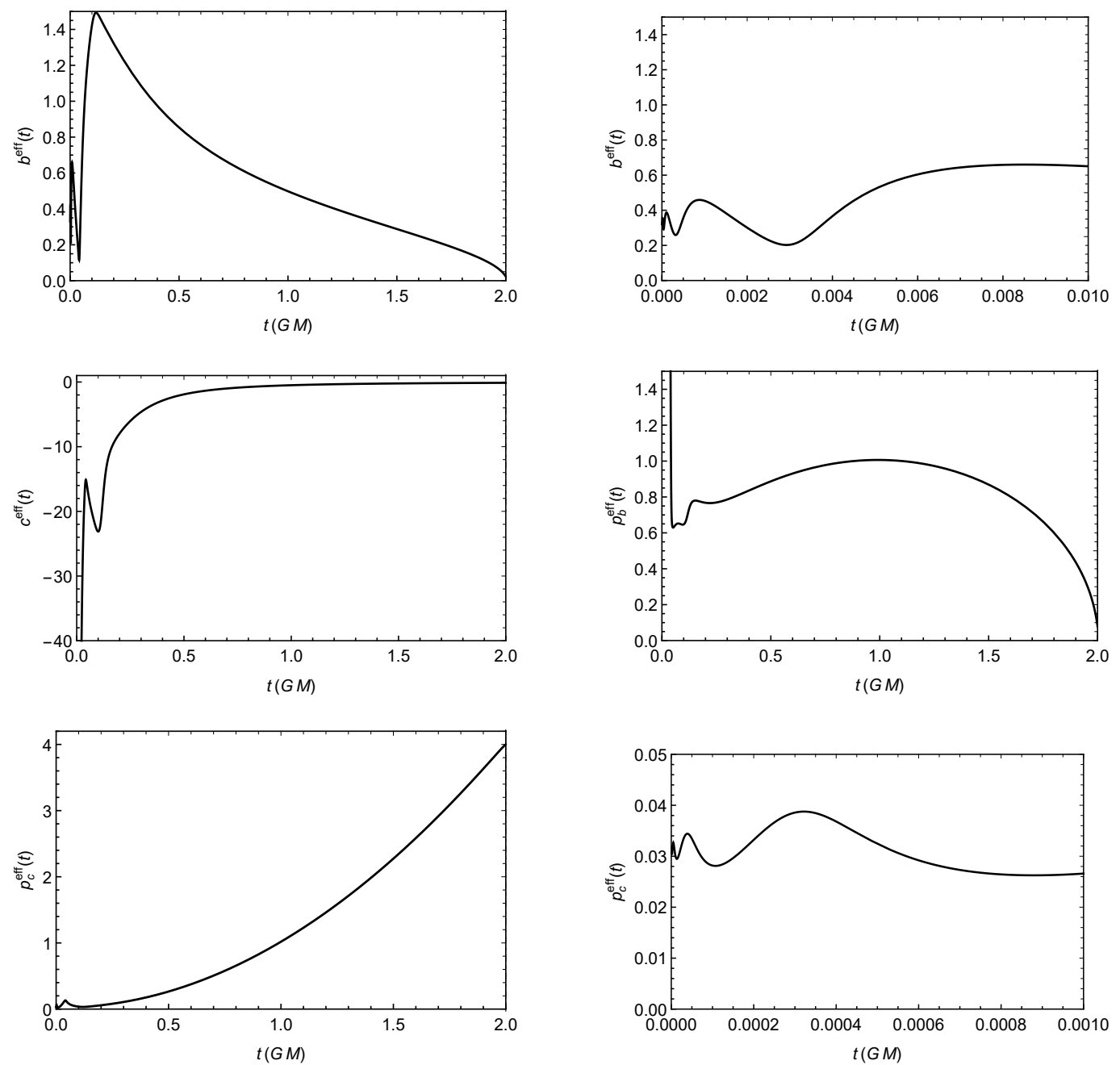

Figure 9. The behavior of the modified solutions in the $\bar{\mu}^{\prime}$ scheme as a function of the Schwarzschild time $t$. The top left figure shows $b(t)$ while top right figure shows the close-up of $b(t)$, close to what used to be a singularity. The middle left figure depicts $c(t)$, and the middle right one shows $p_{b}(t)$. Finally, the behavior of $p_{c}$ and its close-up are depicted in bottom left and bottom right figures, respectively. The figure is plotted using $\gamma=0.5, M=$ $1, G=1, L_{0}=1$, and $\Delta=0.1$.

The equations of motion in this case are

$$
\begin{aligned}
\frac{d b}{d T} & =-\frac{1}{2} \gamma^{2} \frac{\bar{\mu}_{b}^{\prime}}{\sin \left(\bar{\mu}_{b}^{\prime} b\right)}-\frac{1}{2} \frac{\sin \left(\bar{\mu}_{b}^{\prime} b\right)}{\bar{\mu}_{b}^{\prime}}-\frac{p_{c}}{p_{b}}\left[\frac{\sin \left(\bar{\mu}_{c}^{\prime} c\right)}{\bar{\mu}_{c}^{\prime}}+c \cos \left(\bar{\mu}_{c}^{\prime} c\right)\right], \\
\frac{d p_{b}}{d T} & =\frac{1}{2} p_{b} \cos \left(\bar{\mu}_{b}^{\prime} b\right)\left[1-\gamma^{2} \frac{\bar{\mu}_{b}^{\prime 2}}{\sin ^{2}\left(\bar{\mu}_{b}^{\prime} b\right)}\right],
\end{aligned}
$$




$$
\begin{aligned}
\frac{d c}{d T}= & \frac{p_{b}}{2 p_{c}}\left[\gamma^{2} \frac{\bar{\mu}_{b}^{\prime}}{\sin \left(\bar{\mu}_{b}^{\prime} b\right)}\left[1-\frac{\bar{\mu}_{b}^{\prime}}{\sin \left(\bar{\mu}_{b}^{\prime} b\right)} b \cos \left(\bar{\mu}_{b}^{\prime} b\right)\right]-\frac{\sin \left(\bar{\mu}_{b}^{\prime} b\right)}{\bar{\mu}_{b}^{\prime}}\right] \\
& +\frac{b p_{b} \cos \left(\bar{\mu}_{b}^{\prime} b\right)}{2 p_{c}}-\frac{\sin \left(\bar{\mu}_{c}^{\prime} c\right)}{\bar{\mu}_{c}^{\prime}}-c \cos \left(\bar{\mu}_{c}^{\prime} c\right), \\
\frac{d p_{c}}{d T}= & 2 p_{c} \cos \left(\bar{\mu}_{c}^{\prime} c\right) .
\end{aligned}
$$

The behavior of some of these canonical variables as a function of the Schwarzschild time $t$ is now quite different from the previous two schemes. Figure 9 shows the behavior of $b, p_{c}$ and their close-ups near what used to be a singularity as well as the behavior of $c, p_{b}$. Particularly, one notices that $p_{c}$ behaves differently while still remaining nonzero in the interior, hence leading singularity resolution once again. Both $b$ and $p_{c}$ show some sort of damped oscillatory behavior close to the classical singularity which contributes to a more volatile behavior of the Raychaudhuri equation.

The full Raychaudhuri equation in this case takes the following form

$$
\begin{aligned}
\frac{d \theta}{d \tau}= & \frac{1}{\gamma^{2} p_{c}} \frac{\sin ^{2}\left(b \bar{\mu}_{b}^{\prime}\right)}{\bar{\mu}_{b}^{\prime 2}}\left[\cos \left(\bar{\mu}_{b}^{\prime} b\right) \cos \left(\bar{\mu}_{c}^{\prime} c\right)-\frac{\cos ^{2}\left(\bar{\mu}_{b}^{\prime} b\right)}{4}-3 \cos ^{2}\left(\bar{\mu}_{c}^{\prime} c\right)\right] \\
& +\frac{\cos \left(\bar{\mu}_{b}^{\prime} b\right)}{p_{c}}\left[\frac{\cos \left(\bar{\mu}_{b}^{\prime} b\right)}{2}-\cos \left(\bar{\mu}_{c}^{\prime} c\right)-\frac{\gamma^{2}}{4} \cos \left(\bar{\mu}_{b}^{\prime} b\right) \frac{\bar{\mu}_{b}^{\prime 2}}{\sin ^{2}\left(\bar{\mu}_{b}^{\prime} b\right)}\right] .
\end{aligned}
$$

This again looks identical to (4.14) and (4.22), except for the different forms of $\bar{\mu}^{\prime}$ scales compared to previous cases. Before considering the full nonperturbative expression of the above equation, we can check that up to first order in $\Delta$, one obtains

$$
\frac{d \theta}{d \tau} \approx-\frac{1}{2 p_{c}}\left(1+\frac{9 b^{2}}{2 \gamma^{2}}+\frac{\gamma^{2}}{2 b^{2}}\right)+\frac{\Delta}{6 \gamma^{2}}\left[\frac{1}{p_{c}^{2}}\left(3 b^{4}+\gamma^{4}\right)+\frac{3 c^{2}}{p_{b}^{2}}\left(5 b^{2}+\gamma^{2}\right)\right] .
$$

Although this perturbative form of the Raychaudhuri equation is a bit different from previous cases, nevertheless it exhibits the property that the quantum corrections are all positive and hence once again contribute to defocusing of the geodesics.

The full nonperturbative Raychaudhuri equation and its close-ups in this case are plotted in Fig. 10. It is seen that in this scheme, the Raychaudhuri equation exhibits a more volatile behavior and has various bumps particularly when we get closer to where the singularity used to be. Very close to the classical singularity, its form resembles those of $b$ and $p_{c}$, behaving like a damped oscillation.

Two particular features are worth noting in this scheme. First, as we also saw in previous schemes, quantum corrections kick in close to the singularity and dominate the evolution such that the infinite focusing is remedied, hence signaling the resolution of the singularity. Second, this scheme exhibits a nonvanishing value for $\frac{d \theta}{d \tau}$ at or very close to the singularity. In Fig. 10 with the particular choice of numerical values of 

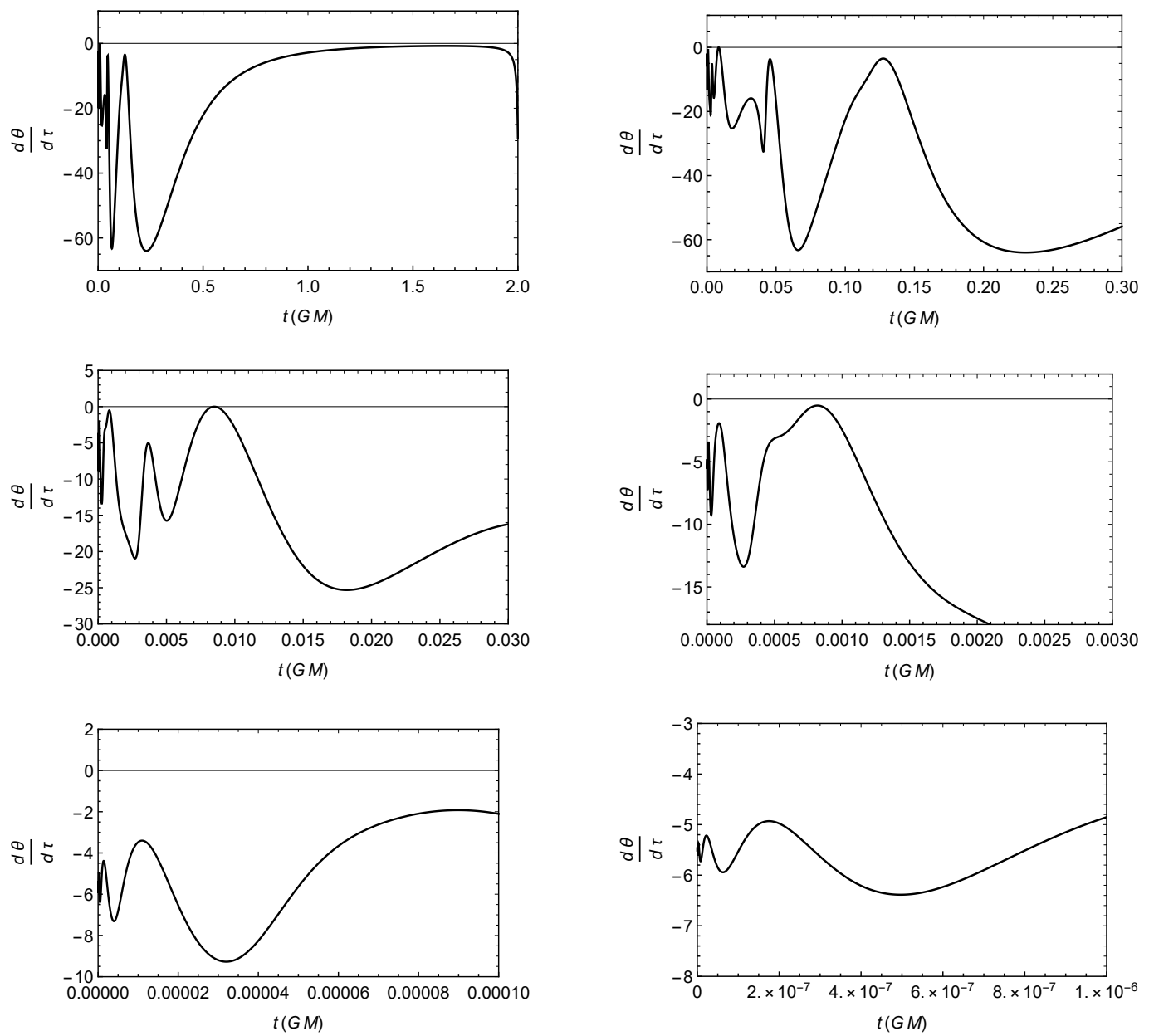

Figure 10. Raychaudhuri equation in the $\bar{\mu}^{\prime}$ scheme. The top left figure shows the behavior over the whole $0 \leq t \leq 2 G M$ range. Other plots show various close-ups of that plot over smaller ranges of $t$. The figure is plotted using $\gamma=0.5, M=1, G=1, L_{0}=1$, and $\Delta=0.1$.

$\gamma, M, G, L_{0}$ and $\Delta$, the value of $\frac{d \theta}{d \tau}$ for $t \rightarrow 0$ is approximately -5.5 . Hence, although a nonvanishing focusing is not achieved in this case at where the singularity used to be, nevertheless, there exists a relatively small focusing.

\section{Discussion and outlook}

In this paper, we have shown that the LQG corrections to the interior of Schwarzschild black hole induce additional terms in the Raychaudhuri equation. Importantly, these terms are repulsive (positive) near the classical singularity of the black hole. This is in contrast to the attractive (negative) terms on the right-hand side of the classical 
Raychaudhuri equation. So, while the former implies the convergence of geodesics, as our explicit computation and related plots show, the quantum generated terms that we estimated are sufficient to negate this convergence. This in turn implies that the primary condition for the Hawking-Penrose singularity theorems to hold true is violated, and the theorems themselves cease to hold. Consequently, geodesics are no longer incomplete, and the classical singularity is resolved. We emphasize that this result is true only for the spherically symmetric Schwarzschild black hole, but we expect it to continue to hold for realistic astrophysical black holes as well, with little or no symmetries. After all, it is the more symmetric solutions which are more likely to demonstrate singularities. While repeating our calculations for the most general black hole singularity may prove technically challenging, we do hope to extend our results to other black hole spacetimes, such as Kerr or Reissner-Nordström. Furthermore, our approach should shed light on cosmological singularities as well. We hope to report on these elsewhere.

\section{Acknowledgments}

This work was supported by the Natural Sciences and Engineering Research Council (NSERC) of Canada.

\section{References}

[1] R. Penrose, Gravitational collapse and space-time singularities, Phys. Rev. Lett. 14 (1965) 57.

[2] S. Hawking and R. Penrose, The Singularities of gravitational collapse and cosmology, Proc. Roy. Soc. Lond. A 314 (1970) 529.

[3] A. Raychaudhuri, Relativistic cosmology. 1., Phys. Rev. 98 (1955) 1123.

[4] S. Das, Quantum Raychaudhuri equation, Phys. Rev. D 89 (2014) 084068 [1311.6539].

[5] D. J. Burger, N. Moynihan, S. Das, S. Shajidul Haque and B. Underwood, Towards the Raychaudhuri Equation Beyond General Relativity, Phys. Rev. D 98 (2018) 024006 [1802.09499].

[6] S. Das, S. S. Haque and B. Underwood, Constraints and horizons for de Sitter with extra dimensions, Phys. Rev. D 100 (2019) 046013 [1905.05864].

[7] T. Thiemann, Modern Canonical Quantum General Relativity, Cambridge Monographs on Mathematical Physics. Cambridge University Press, 2007, 10.1017/CBO9780511755682. 
[8] M. Bojowald, Spherically symmetric quantum geometry: States and basic operators, Class. Quant. Grav. 21 (2004) 3733 [gr-qc/0407017].

[9] A. Ashtekar and M. Bojowald, Quantum geometry and the Schwarzschild singularity, Class. Quant. Grav. 23 (2006) 391 [gr-qc/0509075].

[10] M. Bojowald and R. Swiderski, Spherically symmetric quantum geometry: Hamiltonian constraint, Class. Quant. Grav. 23 (2006) 2129 [gr-qc/0511108].

[11] Böhmer, Christian G. and Vandersloot, Kevin, Loop Quantum Dynamics of the Schwarzschild Interior, Phys. Rev. D 76 (2007) 104030 [0709.2129].

[12] Böhmer, Christian G. and Vandersloot, Kevin, Stability of the Schwarzschild Interior in Loop Quantum Gravity, Phys. Rev. D 78 (2008) 067501 [0807.3042].

[13] A. Corichi and P. Singh, Loop quantization of the Schwarzschild interior revisited, Class. Quant. Grav. 33 (2016) 055006 [1506.08015].

[14] J. Ben Achour, F. Lamy, H. Liu and K. Noui, Non-singular black holes and the Limiting Curvature Mechanism: A Hamiltonian perspective, JCAP 05 (2018) 072 [1712.03876].

[15] A. Ashtekar, J. Olmedo and P. Singh, Quantum extension of the Kruskal spacetime, Phys. Rev. D 98 (2018) 126003 [1806.02406].

[16] J. Ben Achour, F. Lamy, H. Liu and K. Noui, Polymer Schwarzschild black hole: An effective metric, EPL 123 (2018) 20006 [1803.01152].

[17] A. Barrau, K. Martineau and F. Moulin, A status report on the phenomenology of black holes in loop quantum gravity: Evaporation, tunneling to white holes, dark matter and gravitational waves, Universe 4 (2018) 102 [1808.08857].

[18] D. Arruga, J. Ben Achour and K. Noui, Deformed General Relativity and Quantum Black Holes Interior, Universe 6 (2020) 39 [1912.02459].

[19] J. Ben Achour, S. Brahma, S. Mukohyama and J.-P. Uzan, Towards consistent black-to-white hole bounces from matter collapse, JCAP 09 (2020) 020 [2004.12977].

[20] N. Bodendorfer, F. M. Mele and J. Münch, Effective Quantum Extended Spacetime of Polymer Schwarzschild Black Hole, Class. Quant. Grav. 36 (2019) 195015 [1902.04542].

[21] N. Bodendorfer, F. M. Mele and J. Münch, $(b, v)$-Type Variables for Black to White Hole Transitions in Effective Loop Quantum Gravity, 1911.12646.

[22] M. Bojowald and J. D. Reyes, Dilaton Gravity, Poisson Sigma Models and Loop Quantum Gravity, Class. Quant. Grav. 26 (2009) 035018 [0810.5119].

[23] M. Bojowald, T. Harada and R. Tibrewala, Lemaitre-Tolman-Bondi collapse from the perspective of loop quantum gravity, Phys. Rev. D 78 (2008) 064057 [0806.2593].

[24] M. Bojowald and S. Brahma, Signature change in two-dimensional black-hole models of loop quantum gravity, Phys. Rev. D 98 (2018) 026012 [1610.08850]. 
[25] M. Bojowald and S. Brahma, Signature change in loop quantum gravity: Two-dimensional midisuperspace models and dilaton gravity, Phys. Rev. D 95 (2017) 124014 [1610.08840].

[26] M. Bojowald, S. Brahma and D.-h. Yeom, Effective line elements and black-hole models in canonical loop quantum gravity, Phys. Rev. D 98 (2018) 046015 [1803.01119].

[27] S. Brahma, Spherically symmetric canonical quantum gravity, Phys. Rev. D 91 (2015) 124003 [1411.3661].

[28] M. Campiglia, R. Gambini and J. Pullin, Loop quantization of spherically symmetric midi-superspaces : The Interior problem, AIP Conf. Proc. 977 (2008) 52 [0712.0817].

[29] D.-W. Chiou, Phenomenological loop quantum geometry of the Schwarzschild black hole, Phys. Rev. D 78 (2008) 064040 [0807.0665].

[30] A. Corichi, A. Karami, S. Rastgoo and T. Vukašinac, Constraint Lie algebra and local physical Hamiltonian for a generic 2D dilatonic model, Class. Quant. Grav. 33 (2016) 035011 [1508.03036].

[31] J. Cortez, W. Cuervo, H. A. Morales-Técotl and J. C. Ruelas, Effective loop quantum geometry of Schwarzschild interior, Phys. Rev. D 95 (2017) 064041 [1704.03362].

[32] R. Gambini and J. Pullin, Black holes in loop quantum gravity: The Complete space-time, Phys. Rev. Lett. 101 (2008) 161301 [0805.1187].

[33] R. Gambini, J. Pullin and S. Rastgoo, Quantum scalar field in quantum gravity: The vacuum in the spherically symmetric case, Class. Quant. Grav. 26 (2009) 215011 [0906.1774].

[34] R. Gambini, J. Pullin and S. Rastgoo, Quantum scalar field in quantum gravity: the propagator and Lorentz invariance in the spherically symmetric case, Gen. Rel. Grav. 43 (2011) 3569 [1105.0667].

[35] R. Gambini and J. Pullin, Loop quantization of the Schwarzschild black hole, Phys. Rev. Lett. 110 (2013) 211301 [1302.5265].

[36] R. Gambini, J. Olmedo and J. Pullin, Spherically symmetric loop quantum gravity: analysis of improved dynamics, Class. Quant. Grav. 37 (2020) 205012 [2006.01513].

[37] V. Husain and O. Winkler, Quantum resolution of black hole singularities, Class. Quant. Grav. 22 (2005) L127 [gr-qc/0410125].

[38] V. Husain and O. Winkler, Quantum Hamiltonian for gravitational collapse, Phys. Rev. D 73 (2006) 124007 [gr-qc/0601082].

[39] J. G. Kelly, R. Santacruz and E. Wilson-Ewing, Black hole collapse and bounce in effective loop quantum gravity, 2006.09325. 
[40] J. G. Kelly, R. Santacruz and E. Wilson-Ewing, Effective loop quantum gravity framework for vacuum spherically symmetric space-times, 2006.09302.

[41] A. Kreienbuehl, V. Husain and S. S. Seahra, Modified general relativity as a model for quantum gravitational collapse, Class. Quant. Grav. 29 (2012) 095008 [1011.2381].

[42] L. Modesto, Loop quantum black hole, Class. Quant. Grav. 23 (2006) 5587 [gr-qc/0509078].

[43] L. Modesto and I. Premont-Schwarz, Self-dual Black Holes in LQG: Theory and Phenomenology, Phys. Rev. D 80 (2009) 064041 [0905.3170].

[44] J. Olmedo, S. Saini and P. Singh, From black holes to white holes: a quantum gravitational, symmetric bounce, Class. Quant. Grav. 34 (2017) 225011 [1707.07333].

[45] T. Thiemann and H. Kastrup, Canonical quantization of spherically symmetric gravity in Ashtekar's selfdual representation, Nucl. Phys. B 399 (1993) 211 [gr-qc/9310012].

[46] C. Zhang, Y. Ma, S. Song and X. Zhang, Loop quantum Schwarzschild interior and black hole remnant, Phys. Rev. D 102 (2020) 041502 [2006.08313].

[47] J. Ziprick, J. Gegenberg and G. Kunstatter, Polymer Quantization of a Self-Gravitating Thin Shell, Phys. Rev. D 94 (2016) 104076 [1609.06665].

[48] M. Campiglia, R. Gambini and J. Pullin, Loop quantization of spherically symmetric midi-superspaces, Class. Quant. Grav. 24 (2007) 3649 [gr-qc/0703135].

[49] R. Gambini, J. Pullin and S. Rastgoo, New variables for $1+1$ dimensional gravity, Class. Quant. Grav. 27 (2010) 025002 [0909.0459].

[50] S. Rastgoo, A local true Hamiltonian for the CGHS model in new variables, 1304.7836.

[51] A. Corichi, J. Olmedo and S. Rastgoo, Callan-Giddings-Harvey-Strominger vacuum in loop quantum gravity and singularity resolution, Phys. Rev. D 94 (2016) 084050 [1608.06246].

[52] H. A. Morales-Técotl, S. Rastgoo and J. C. Ruelas, Effective dynamics of the Schwarzschild black hole interior with inverse triad corrections, 1806.05795.

[53] J. Ben Achour, S. Brahma and J.-P. Uzan, Bouncing compact objects. Part I. Quantum extension of the Oppenheimer-Snyder collapse, JCAP 03 (2020) 041 [2001.06148].

[54] J. Ben Achour and J.-P. Uzan, Bouncing compact objects II: Effective theory of a pulsating Planck star, 2001.06153.

[55] A. Ashtekar, T. Pawlowski and P. Singh, Quantum nature of the big bang, Phys. Rev. Lett. 96 (2006) 141301 [gr-qc/0602086].

[56] A. Ashtekar, T. Pawlowski and P. Singh, Quantum Nature of the Big Bang: An Analytical and Numerical Investigation. I., Phys. Rev. D 73 (2006) 124038 [gr-qc/0604013]. 
[57] A. Ashtekar, S. Fairhurst and J. L. Willis, Quantum gravity, shadow states, and quantum mechanics, Class. Quant. Grav. 20 (2003) 1031 [gr-qc/0207106].

[58] A. Corichi, T. Vukasinac and J. A. Zapata, Polymer Quantum Mechanics and its Continuum Limit, Phys. Rev. D 76 (2007) 044016 [0704.0007].

[59] H. A. Morales-Técotl, S. Rastgoo and J. C. Ruelas, Path integral polymer propagator of relativistic and nonrelativistic particles, Phys. Rev. D 95 (2017) 065026 [1608.04498].

[60] H. A. Morales-Técotl, D. H. Orozco-Borunda and S. Rastgoo, Polymer quantization and the saddle point approximation of partition functions, Phys. Rev. D 92 (2015) 104029 [1507.08651].

[61] E. Flores-González, H. A. Morales-Técotl and J. D. Reyes, Propagators in Polymer Quantum Mechanics, Annals Phys. 336 (2013) 394 [1302.1906].

[62] E. Alesci, S. Bahrami and D. Pranzetti, Quantum evolution of black hole initial data sets: Foundations, Phys. Rev. D 98 (2018) 046014 [1807.07602].

[63] E. Alesci, S. Bahrami and D. Pranzetti, Quantum gravity predictions for black hole interior geometry, Phys. Lett. B 797 (2019) 134908 [1904.12412].

[64] E. Alesci, S. Bahrami and D. Pranzetti, Asymptotically de Sitter universe inside a Schwarzschild black hole, Phys. Rev. D 102 (2020) 066010 [2007.06664].

[65] A. Saini and D. Stojkovic, Nonlocal (but also nonsingular) physics at the last stages of gravitational collapse, Phys. Rev. D 89 (2014) 044003 [1401.6182].

[66] E. Greenwood and D. Stojkovic, Quantum gravitational collapse: Non-singularity and non-locality, JHEP 06 (2008) 042 [0802.4087].

[67] J. E. Wang, E. Greenwood and D. Stojkovic, Schrodinger formalism, black hole horizons and singularity behavior, Phys. Rev. D 80 (2009) 124027 [0906.3250].

[68] C. Collins, Global structure of the Kantowski-Sachs cosmological models, J. Math. Phys. 18 (1977) 2116.

[69] D.-W. Chiou, Phenomenological dynamics of loop quantum cosmology in Kantowski-Sachs spacetime, Phys. Rev. D 78 (2008) 044019 [0803.3659].

[70] J. von Neumann, Die eindeutigkeit der Schrödingerschen operatoren, Math. Ann. 104 (1931) 570 .

[71] H. A. Morales-Técotl, D. H. Orozco-Borunda and S. Rastgoo, Polymerization, the Problem of Access to the Saddle Point Approximation, and Thermodynamics, in 14th Marcel Grossmann Meeting on Recent Developments in Theoretical and Experimental General Relativity, Astrophysics, and Relativistic Field Theories, vol. 4, pp. 4054-4059, World Scientific, Singapore, 2017, DOI [1603.08076]. 
[72] L. Modesto, Semiclassical loop quantum black hole, Int. J. Theor. Phys. 49 (2010) 1649 [0811.2196].

[73] A. Joe and P. Singh, Kantowski-Sachs spacetime in loop quantum cosmology: bounds on expansion and shear scalars and the viability of quantization prescriptions, Class.

Quant. Grav. 32 (2015) 015009 [1407.2428]. 